\title{
Convergence of Wachspress coordinates: from polygons to curved domains
}

\author{
Jiří Kosinka \\ Computer Laboratory \\ University of Cambridge \\ Jiri.Kosinka@cl.cam.ac.uk
}

\author{
Michael Bartoň \\ Numerical Porous Media Center \\ King Abdullah University of Science and Technology \\ Michael.Barton@kaust.edu.sa
}

\begin{abstract}
Given a smooth, strictly convex planar domain, we investigate point-wise convergence of the sequence of Wachspress coordinates defined over finer and finer inscribed polygonal approximations of the domain. Based on a relation between the discrete Wachspress case and the limit smooth case given by the Wachspress kernel defined by Warren et al., we show that the corresponding sequences of Wachspress interpolants and mappings converge as $\mathcal{O}\left(h^{2}\right)$ for a sampling step size $h$ of the boundary curve of the domain as $h \rightarrow 0$. Several examples are shown to numerically validate the results and to visualise the behaviour of discrete interpolants and mappings as they converge to their smooth counterparts. Empirically, the same convergence order is observed also for mean value coordinates. Moreover, our numerical tests suggest that the convergence of interpolants and mappings is uniform both in the Wachspress and mean value cases.
\end{abstract}

Keywords: Barycentric coordinates, Barycentric mapping, Interpolation, Convergence

\section{Introduction}

Generalised barycentric coordinates play an important role in geometric modelling and computer graphics. Their applications include shading, shape deformations [10,16], image morphing [11], and colour blending. These coordinates are a generalisation of the standard barycentric coordinates for triangles to polygons. Barycentric coordinates for triangles are unique, but that is no longer the case for coordinates for polygons with more than three vertices. Popular coordinates for polygons include Wachspress coordinates [14], mean value coordinates [4], and harmonic coordinates [9].

Polygonal coordinates have been generalised to so-called barycentric kernels for smooth domains. Examples of such kernels are the kernel defined by Warren et al. [15], which we refer to as the Wachspress kernel, and the mean value kernel [1].

Since a smooth domain in $\mathbb{R}^{2}$ can be obtained as the limit of a convergent sequence of polygons (see Fig. 1), natural questions arise: Does the sequence of barycentric coordinates for the finer and finer polygons converge to the kernel of the limit, i.e., the smooth domain? What is the order and type of that convergence and does it apply also to barycentric interpolants and mappings? This paper investigates these questions.

We first review generalised barycentric coordinates for polygons in Section 2 and barycentric kernels for smooth domains in Section 3. These coordinates and kernels give rise to barycentric interpolants and mappings (Section 4). The convergence of Wachspress coordinates for a convergent sequence of polygons is studied in Section 5. Our convergence results for coordinates carry over to barycentric interpolants and mappings. Extensions of our results for Wachspress coordinates to mean value coordinates are discussed in Section 6. The paper is concluded by several numerical examples.

\section{Barycentric coordinates on polygons}

Let $P$ be a strictly convex polygon in the plane with vertices $\mathbf{p}_{1}, \mathbf{p}_{2}, \ldots, \mathbf{p}_{n}, n \geq 3$, ordered anticlockwise. We regard $P$ as an open set and denote $\bar{P}$ its closure. Functions $\lambda_{i}: \bar{P} \rightarrow \mathbb{R}, i=1, \ldots, n$ form a set of barycentric coordinates on $P$ if the following three properties are satisfied for all $\mathrm{x} \in \bar{P}$ :

1. Non-negativity

$$
\lambda_{i}(\mathbf{x}) \geq 0, \quad i=1, \ldots, n
$$

2. Partition of unity

$$
\sum_{i=1}^{n} \lambda_{i}(\mathbf{x})=1
$$



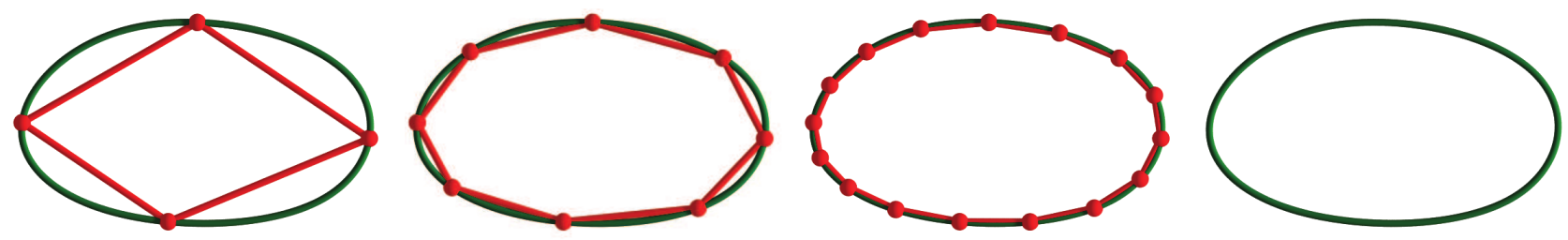

Figure 1: A sequence of polygons (red) converging to a smooth domain (green).

\section{Barycentric property}

$$
\sum_{i=1}^{n} \lambda_{i}(\mathbf{x}) \mathbf{p}_{i}=\mathbf{x}
$$

For $n=3$, the triple $\lambda_{1}, \lambda_{2}$, and $\lambda_{3}$ are the unique barycentric coordinates for triangles. For $n \geq 4$, the set of (generalised) barycentric coordinates is not unique any more. The most popular choices of barycentric coordinates for convex polygons are Wachspress coordinates [14], mean value coordinates [4], and harmonic coordinates [9]. The latter two extend to non-convex polygons.

As shown in [3], all continuous barycentric coordinates are piece-wise linear at $\partial P$ and satisfy the Lagrange property

$$
\lambda_{i}\left(\mathbf{p}_{j}\right)=\delta_{i, j}
$$

Barycentric coordinates are typically defined in terms of weight functions. Each vertex $\mathbf{p}_{i}$ of $P$ is assigned a weight function $w_{i}(\mathbf{x})$. These weights are then normalised to obtain the barycentric coordinates

$$
\lambda_{i}(\mathbf{x})=\frac{w_{i}(\mathbf{x})}{W}, \quad W=\sum_{j=1}^{n} w_{j}(\mathbf{x}) ; \quad \mathbf{x} \in \bar{P} .
$$

For non-negative weights, this construction guarantees that the functions $\lambda_{i}(\mathbf{x})$ are non-negative (1) and form a partition of unity (2). The barycentric property (3) has to be proved separately.

For later use, we introduce the following notation. Let $\mathcal{D}(\mathbf{a}, \mathbf{b}, \mathbf{c})$ denote the determinant

$$
\mathcal{D}(\mathbf{a}, \mathbf{b}, \mathbf{c})=\left|\begin{array}{ccc}
1 & 1 & 1 \\
a^{1} & b^{1} & c^{1} \\
a^{2} & b^{2} & c^{2}
\end{array}\right|=(\mathbf{b}-\mathbf{a}) \times(\mathbf{c}-\mathbf{b}),
$$

in which $\mathbf{a}=\left(a^{1}, a^{2}\right), \mathbf{b}=\left(b^{1}, b^{2}\right), \mathbf{c}=\left(c^{1}, c^{2}\right)$, and $\mathbf{a} \times \mathbf{b}$ denotes the planar cross product $a^{1} b^{2}-a^{2} b^{1}$. The signed area of the triangle $\triangle(\mathbf{a}, \mathbf{b}, \mathbf{c})$ is then $\mathcal{D}(\mathbf{a}, \mathbf{b}, \mathbf{c}) / 2$. Further, we set

$$
A_{i}(\mathbf{x})=\mathcal{D}\left(\mathbf{x}, \mathbf{p}_{i}, \mathbf{p}_{i+1}\right), B_{i}(\mathbf{x})=\mathcal{D}\left(\mathbf{x}, \mathbf{p}_{i-1}, \mathbf{p}_{i+1}\right), C_{i}=\mathcal{D}\left(\mathbf{p}_{i-1}, \mathbf{p}_{i}, \mathbf{p}_{i+1}\right)
$$

see Fig. 2. Here and throughout the paper, the indices of $A_{i}, B_{i}, \mathbf{p}_{i}$ and so on are treated cyclically with respect to $n$.

\subsection{Wachspress coordinates}

Wachspress coordinates are defined by the weights

$$
w_{i}(\mathbf{x})=C_{i} \prod_{j \neq i-1, i} A_{j}(\mathbf{x}) .
$$

Since $A_{j}$ is linear in $\mathbf{x}$, it follows that $w_{i}$ is a polynomial of degree $n-2$. Moreover, Wachspress coordinates are $C^{\infty}$ over $\bar{P}$. They are rational in the coordinates $x^{1}$ and $x^{2}$ of the point $\mathbf{x}=\left(x^{1}, x^{2}\right)$ with the degree of the numerator at most $n-2$ and the degree of the denominator, $W$, at most $n-3$; see (5). The barycentric property of Wachspress coordinates was established in [12].

For points that do not lie on the boundary of $P$, the weights of (8) give the same coordinates as the weights

$$
w_{i}(\mathbf{x})=\frac{C_{i}}{A_{i-1} A_{i}} .
$$

We use this more convenient expression in our convergence analysis in Section 5. 


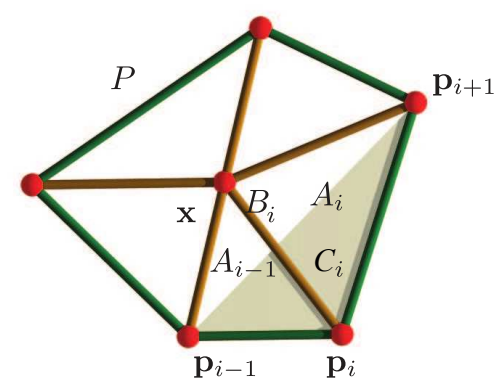

Figure 2: Determinants involved in the definition of Wachspress coordinates. The $i$-th weight $w_{i}$ of $\mathbf{x}$ in a polygon $P$ depends only on three consecutive vertices $\mathbf{p}_{i-1}, \mathbf{p}_{i}$, and $\mathbf{p}_{i+1}$.

\section{Barycentric kernels on curved domains}

So far we have discussed barycentric coordinates for polygons, but barycentric coordinates are also defined for domains with curved boundaries. Such coordinates are typically called barycentric kernels.

Let $\Omega$ be a bounded, open, strictly convex region of $\mathbb{R}^{2}$ and let $\mathbf{p}:[a, b] \rightarrow \mathbb{R}^{2}$, with $\mathbf{p}(a)=\mathbf{p}(b)$, be a smooth continuous parametrisation of $\partial \Omega$. To avoid pathological cases we assume that $\mathbf{p}$ is injective in $[a, b)$.

A kernel $\lambda$ is called barycentric if for all $\mathbf{x} \in \Omega$, it satisfies:

1. Non-negativity

$$
\lambda(\mathbf{x}, t) \geq 0, \quad t \in[a, b)
$$

2. Partition of unity

$$
\int_{a}^{b} \lambda(\mathbf{x}, t) \mathrm{d} t=1
$$

3. Barycentric property

$$
\int_{a}^{b} \lambda(\mathbf{x}, t) \mathbf{p}(t) \mathrm{d} t=\mathbf{x} .
$$

Note that the latter two equations are integral equivalents of properties (2) and (3) for barycentric coordinates defined on polygons.

\subsection{The Wachspress kernel}

Again, as in the discrete case for polygons, barycentric kernels are typically defined using weights

$$
\lambda(\mathbf{x}, t)=w(\mathbf{x}, t) / \int_{a}^{b} w(\mathbf{x}, s) \mathrm{d} s .
$$

The Wachspress kernel is given by

$$
w(\mathbf{x}, t)=\frac{\mathbf{p}^{\prime}(t) \times \mathbf{p}^{\prime \prime}(t)}{\left((\mathbf{p}(t)-\mathbf{x}) \times \mathbf{p}^{\prime}(t)\right)^{2}} .
$$

This is a special case of a general formula for barycentric kernels in any dimension introduced by Warren et al. [15].

At the boundary, i.e., when $\mathbf{x}=\mathbf{p}(u)$ for some parameter value $u \in[a, b]$, the value of $\lambda(\mathbf{p}(u), t)$ is given by the Dirac delta function, namely $\lambda(\mathbf{p}(u), t)=\delta(u-t)$. The parametrisation $\mathbf{p}(t)$ is assumed to be at least $C^{2}$.

\section{Barycentric interpolation and mappings}

Barycentric coordinates and kernels can be used to solve boundary interpolation problems and also to perform deformations. Here we summarise some basic results on barycentric interpolation and mappings. 

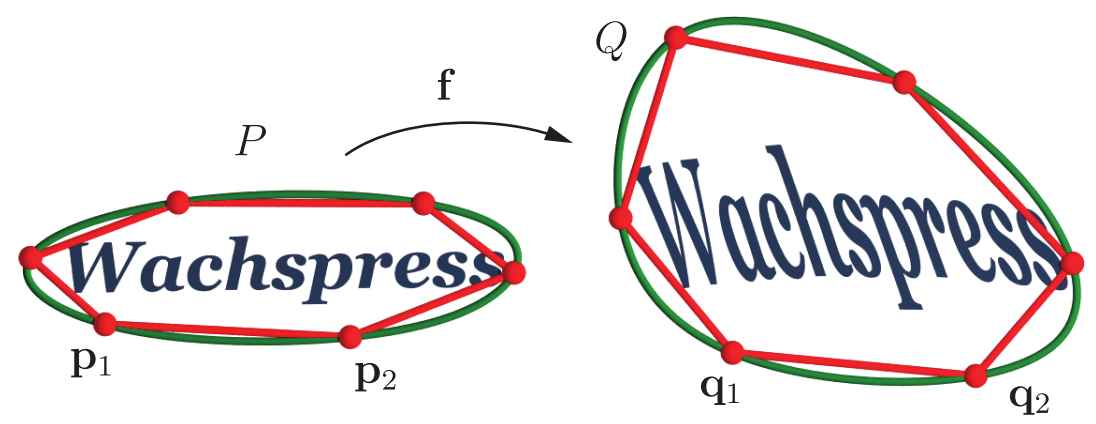

Figure 3: A barycentric mapping between two hexagons $P$ and $Q$.

\subsection{Barycentric interpolation}

In the discrete case of barycentric coordinates $\lambda_{i}$ for a polygon $P$, let a function $f$ be known at the vertices $\mathbf{p}_{i}$ of $P$. Then the function $g: \bar{P} \rightarrow \mathbb{R}$, given by

$$
g(\mathbf{x})=\sum_{i=1}^{n} \lambda_{i}(\mathbf{x}) f\left(\mathbf{p}_{i}\right),
$$

interpolates $f$. This is an immediate consequence of the Lagrange property (4).

Similarly, in the smooth case of a barycentric kernel $\lambda$, for a continuous function $f$ defined on the boundary of $\Omega$, the function $g: \bar{\Omega} \rightarrow \mathbb{R}$

$$
g(\mathbf{x})=\int_{a}^{b} \lambda(\mathbf{x}, t) f(\mathbf{p}(t)) \mathrm{d} t
$$

interpolates $f$. This interpolation property was shown in [6].

\subsection{Barycentric mappings}

Consider first the discrete case. Let $Q \subset \mathbb{R}^{2}$ be a second polygon with vertices $\mathbf{q}_{1}, \ldots, \mathbf{q}_{n}$. A barycentric mapping f from $\bar{P}$ to $\bar{Q}$ is defined via barycentric coordinates $\lambda_{i}$ for $P$ as

$$
\mathbf{f}(\mathbf{x})=\sum_{i=1}^{n} \lambda_{i}(\mathbf{x}) \mathbf{q}_{i} .
$$

From the properties of $\lambda_{i}$ it follows that $\mathbf{f}(\bar{P}) \subset \bar{Q}$ and that $\mathbf{f}$ maps $\partial P$ to $\partial Q$ in a piece-wise linear fashion; in particular $\mathbf{f}\left(\mathbf{p}_{i}\right)=\mathbf{q}_{i}$ for all $i$. The injectivity of this mapping between strictly convex polygons induced by Wachspress coordinates was established in [5]. The injectivity of composite mean value mappings was recently studied in [13].

In the smooth case of a barycentric kernel $\lambda$ for a domain $\Omega$, let $\Psi \subset \mathbb{R}^{2}$ be a second domain and $\mathbf{q}:[a, b] \rightarrow \mathbb{R}^{2}$ an injective parametrisation in $[a, b)$, with $\mathbf{q}(a)=\mathbf{q}(b)$. A barycentric mapping $\mathbf{f}: \Omega \rightarrow \mathbb{R}^{2}$ is given by

$$
\mathbf{f}(\mathbf{x})=\int_{a}^{b} \lambda(\mathbf{x}, t) \mathbf{q}(t) \mathrm{d} t .
$$

This mapping, due to the interpolation property of $\lambda(\mathbf{x}, t)$, continuously extends to the boundary of $\Omega$. Similarly to the discrete case, it was shown in [6] that the Wachspress kernel gives rise to injective mappings between convex domains.

\section{Convergence}

In this section we investigate the relation of the Wachspress kernel defined over a smooth convex domain $\Omega$ to Wachspress coordinates defined over a convergent sequence of inscribed polygonal approximations of $\Omega$; see Fig. 1. 


\subsection{Wachspress coordinates and kernels}

We approximate the curve $\mathbf{p}(t)$, the boundary curve of $\Omega$, by a sequence of polygons $P_{h}$. For any sample of parameter values $a<t_{1}<t_{2}<\cdots<t_{n} \leq b$, define $t_{0}:=t_{n}$ and $t_{n+1}:=t_{1}$, and let $h_{j}=t_{j+1}-t_{j}$, and $h=\max _{j=1, \ldots, n} h_{j}$. Let $P_{h}$ denote the convex polygon with vertices $\mathbf{p}_{i}=\mathbf{p}\left(t_{i}\right), i=1, \ldots, n$.

If we define the first order divided difference

$$
\mathbf{p}_{j}^{[1]}=\frac{\mathbf{p}_{j+1}-\mathbf{p}_{j}}{h_{j}}
$$

we can write the Wachspress weight function $w_{i}(\mathbf{x})$ as

$$
w_{i}(\mathbf{x})=\frac{b_{i}}{c_{i}(\mathbf{x})},
$$

where

$$
b_{i}=\mathbf{p}_{i-1}^{[1]} \times \mathbf{p}_{i}^{[1]} \quad \text { and } \quad c_{i}(\mathbf{x})=\left(\left(\mathbf{p}_{i}-\mathbf{x}\right) \times \mathbf{p}_{i-1}^{[1]}\right)\left(\left(\mathbf{p}_{i}-\mathbf{x}\right) \times \mathbf{p}_{i}^{[1]}\right) .
$$

In the following, we assume that $\mathbf{x} \in \Omega$ and $h$ is small enough that $\mathbf{x} \in P_{h}$. As no confusion is likely to arise, we do not make the dependence of $w_{i}(\mathbf{x})$ and $\lambda_{i}(\mathbf{x})$ on the sampling explicit.

Lemma 5.1 If $\mathbf{p} \in C^{4}[a, b]$, there is a function $e(\mathbf{x}, t)$, with $e(\mathbf{x}, \cdot) \in C^{1}[a, b]$ such that

$$
w_{i}(\mathbf{x})=k_{i}\left(w\left(\mathbf{x}, t_{i}\right)+\left(h_{i}-h_{i-1}\right) e\left(\mathbf{x}, t_{i}\right)\right)+R_{i},
$$

where

$$
k_{i}=\frac{h_{i-1}+h_{i}}{2}=\frac{t_{i+1}-t_{i-1}}{2}
$$

and there is some function $C=C(\mathbf{x})$, independent of $i$, such that $\left|R_{i}\right| \leq C k_{i}^{3}$.

Proof. Since $\mathbf{p} \in C^{4}[a, b]$, the Taylor expansion gives

$$
\begin{aligned}
& \mathbf{p}_{i+1}=\mathbf{p}_{i}+h_{i} \mathbf{p}_{i}^{\prime}+\frac{h_{i}^{2}}{2} \mathbf{p}_{i}^{\prime \prime}+\frac{h_{i}^{3}}{6} \mathbf{p}_{i}^{\prime \prime \prime}+\mathcal{O}\left(h_{i}^{4}\right), \\
& \mathbf{p}_{i-1}=\mathbf{p}_{i}-h_{i-1} \mathbf{p}_{i}^{\prime}+\frac{h_{i-1}^{2}}{2} \mathbf{p}_{i}^{\prime \prime}-\frac{h_{i-1}^{3}}{6} \mathbf{p}_{i}^{\prime \prime \prime}+\mathcal{O}\left(h_{i-1}^{4}\right),
\end{aligned}
$$

and so

$$
\begin{gathered}
\mathbf{p}_{i}^{[1]}=\mathbf{p}_{i}^{\prime}+\frac{h_{i}}{2} \mathbf{p}_{i}^{\prime \prime}+\frac{h_{i}^{2}}{6} \mathbf{p}_{i}^{\prime \prime \prime}+\mathcal{O}\left(h_{i}^{3}\right), \\
\mathbf{p}_{i-1}^{[1]}=\mathbf{p}_{i}^{\prime}-\frac{h_{i-1}}{2} \mathbf{p}_{i}^{\prime \prime}+\frac{h_{i-1}^{2}}{6} \mathbf{p}_{i}^{\prime \prime \prime}+\mathcal{O}\left(h_{i-1}^{3}\right) .
\end{gathered}
$$

Then,

$$
b_{i}=k_{i}\left(\mathbf{p}_{i}^{\prime} \times \mathbf{p}_{i}^{\prime \prime}+\frac{h_{i}-h_{i-1}}{3} \mathbf{p}_{i}^{\prime} \times \mathbf{p}_{i}^{\prime \prime \prime}\right)+\mathcal{O}\left(k_{i}^{3}\right),
$$

and, with $\mathbf{d}(\mathbf{x}, t):=\mathbf{p}(t)-\mathbf{x}$, and $\mathbf{d}_{i}:=\mathbf{d}\left(\mathbf{x}, t_{i}\right)$,

$$
c_{i}(\mathbf{x})=\left(\mathbf{d}_{i} \times \mathbf{p}_{i}^{\prime}\right)^{2}+\frac{h_{i}-h_{i-1}}{2}\left(\mathbf{d}_{i} \times \mathbf{p}_{i}^{\prime}\right)\left(\mathbf{d}_{i} \times \mathbf{p}_{i}^{\prime \prime}\right)+\mathcal{O}\left(k_{i}^{2}\right)
$$

and therefore, as $\left(a_{0}+a_{1} h+\mathcal{O}\left(h^{2}\right)\right)\left(\frac{1}{a_{0}}-\frac{a_{1}}{a_{0}^{2}} h+\mathcal{O}\left(h^{2}\right)\right)=1+\mathcal{O}\left(h^{2}\right)$ for $a_{0} \neq 0$,

$$
\frac{1}{c_{i}(\mathbf{x})}=\frac{1}{\left(\mathbf{d}_{i} \times \mathbf{p}_{i}^{\prime}\right)^{2}}-\frac{h_{i}-h_{i-1}}{2} \frac{\mathbf{d}_{i} \times \mathbf{p}_{i}^{\prime \prime}}{\left(\mathbf{d}_{i} \times \mathbf{p}_{i}^{\prime}\right)^{3}}+\mathcal{O}\left(k_{i}^{2}\right) .
$$

Multiplying (19) by (20) gives the result, with

$$
e(\mathbf{x}, t):=\frac{\left(\mathbf{p}^{\prime}(t) \times \mathbf{p}^{\prime \prime \prime}(t)\right)}{3\left(\mathbf{d}(\mathbf{x}, t) \times \mathbf{p}^{\prime}(t)\right)^{2}}-\frac{\left(\mathbf{p}^{\prime}(t) \times \mathbf{p}^{\prime \prime}(t)\right)\left(\mathbf{d}(\mathbf{x}, t) \times \mathbf{p}^{\prime \prime}(t)\right)}{2\left(\mathbf{d}(\mathbf{x}, t) \times \mathbf{p}^{\prime}(t)\right)^{3}} .
$$

Since $\mathbf{p} \in C^{4}[a, b]$ it follows that $e(\mathbf{x}, \cdot) \in C^{1}[a, b]$. 
To establish convergence orders of barycentric interpolants and mappings, we next establish a relationship between $\sum_{i=1}^{n} w_{i}(\mathbf{x})$ in the discrete case and $\int_{a}^{b} w(\mathbf{x}, t) \mathrm{d} t$ in the smooth case. These expressions are linked by the trapezoidal rule. More precisely, we derive the following

Lemma 5.2 For $\mathbf{x} \in \Omega$ and for $u \in C^{2}[a, b]$ and $\mathbf{p} \in C^{4}[a, b]$,

$$
\sum_{i=1}^{n} w_{i}(\mathbf{x}) u\left(t_{i}\right)=\int_{a}^{b} w(\mathbf{x}, t) u(t) \mathrm{d} t+\mathcal{O}\left(h^{2}\right) \quad \text { as } \quad h \rightarrow 0 .
$$

Proof. With $\mathbf{x}$ fixed, let $\tilde{w}(t)=w(\mathbf{x}, t) u(t)$ and $\tilde{e}(t)=e(\mathbf{x}, t) u(t)$. Then, applying Lemma 5.1 gives

$$
\sum_{i=1}^{n} w_{i}(\mathbf{x}) u\left(t_{i}\right)=D_{1}+D_{2}+D_{3}
$$

where

$$
D_{1}=\frac{1}{2} \sum_{i=1}^{n}\left(h_{i-1}+h_{i}\right) \tilde{w}\left(t_{i}\right)=\frac{1}{2} \sum_{i=1}^{n} h_{i}\left(\tilde{w}\left(t_{i}\right)+\tilde{w}\left(t_{i+1}\right)\right)
$$

and

$$
D_{2}=\frac{1}{2} \sum_{i=1}^{n}\left(h_{i}^{2}-h_{i-1}^{2}\right) \tilde{e}\left(t_{i}\right)=\frac{1}{2} \sum_{i=1}^{n} h_{i}^{2}\left(\tilde{e}\left(t_{i}\right)-\tilde{e}\left(t_{i+1}\right)\right),
$$

and

$$
D_{3}=\sum_{i=1}^{n} R_{i} u\left(t_{i}\right)
$$

Consider first the term $D_{1}$. Since $\mathbf{p} \in C^{4}[a, b], w(\mathbf{x}, \cdot) \in C^{2}[a, b]$, and so $\tilde{w}$ belongs to $C^{2}[a, b]$ as well. Therefore, since $D_{1}$ is the composite trapezoidal rule for $\tilde{w}$,

$$
D_{1}=\int_{a}^{b} \tilde{w}(t) \mathrm{d} t+\mathcal{O}\left(h^{2}\right) .
$$
gives

Consider next $D_{2}$. Since $e(\mathbf{x}, \cdot)$ and $u$ are both in $C^{1}[a, b]$, it follows that $\tilde{e} \in C^{1}[a, b]$. Thus, the mean value theorem

$$
D_{2}=-\frac{1}{2} \sum_{i=1}^{n} h_{i}^{3} \tilde{e}^{\prime}\left(\xi_{i}\right)
$$

where $\xi_{i}$ is some point in $\left(t_{i}, t_{i+1}\right)$. It follows that with $\|\cdot\|$ the max norm on $[a, b]$,

$$
\left|D_{2}\right| \leq \frac{1}{2} \sum_{i=1}^{n} h_{i}^{3}\left\|\tilde{e}^{\prime}\right\| \leq \frac{1}{2} \sum_{i=1}^{n} h_{i} h^{2}\left\|\tilde{e}^{\prime}\right\|=\frac{1}{2}(b-a) h^{2}\left\|\tilde{e}^{\prime}\right\| .
$$

Finally we treat $D_{3}$ :

$$
D_{3} \leq \sum_{i=1}^{n}\left|R_{i}\left\|u\left(t_{i}\right) \mid \leq C\right\| u\left\|\sum_{i=1}^{n} k_{i}^{3} \leq C\right\| u\left\|\sum_{i=1}^{n} h_{i} h^{2}=C\right\| u \|(b-a) h^{2} .\right.
$$

Since both $D_{2}$ and $D_{3}$ are $\mathcal{O}\left(h^{2}\right)$, this completes the proof.

Note that when the points $t_{i}$ are uniformly spaced, $k_{i}=h$ and $h_{i}-h_{i-1}=0$ and Lemma 5.1 implies that

$$
w_{i}(\mathbf{x})=h w\left(\mathbf{x}, t_{i}\right)+\mathcal{O}\left(h^{3}\right) .
$$

Combined with Lemma 5.2, one obtains

$$
\lambda_{i}(\mathbf{x})=h \lambda\left(\mathbf{x}, t_{i}\right)+\mathcal{O}\left(h^{3}\right) .
$$


Remark 5.1 We emphasise that in the analysis above, $C=C(\mathbf{x})$ is independent of $i$, but it depends on $\mathbf{x}$. By considering more terms in the expansion of $w_{i}(\mathbf{x})$ in the case of uniform sampling, one obtains

$$
w_{i}(\mathbf{x})=h w\left(\mathbf{x}, t_{i}\right)+h^{3} d\left(\mathbf{x}, t_{i}\right)+\mathcal{O}\left(h^{4}\right)
$$

with

$$
d\left(\mathbf{x}, t_{i}\right)=\frac{\mathbf{p}_{i}^{\prime} \times \mathbf{p}_{i}^{\prime \prime}}{12\left(\mathbf{d}_{i} \times \mathbf{p}_{i}^{\prime}\right)^{4}}\left(4\left(\mathbf{d}_{i} \times \mathbf{p}_{i}^{\prime}\right)\left(\mathbf{d}_{i} \times \mathbf{p}_{i}^{\prime \prime \prime}\right)-9\left(\mathbf{d}_{i} \times \mathbf{p}_{i}^{\prime \prime}\right)^{2}\right)+\frac{\mathbf{p}_{i}^{\prime} \times \mathbf{p}_{i}^{\prime \prime \prime \prime}+2 \mathbf{p}_{i}^{\prime \prime} \times \mathbf{p}_{i}^{\prime \prime \prime}}{12\left(\mathbf{d}_{i} \times \mathbf{p}_{i}^{\prime}\right)^{2}} .
$$

The coefficient $d\left(\mathbf{x}, t_{i}\right)$ of $h^{3}$ diverges when $\mathbf{x}$ tends to the point $\mathbf{p}\left(t_{i}\right)$ on the boundary of $P_{h}$ (and $\Omega$ ). Recall that $w\left(\mathbf{x}, t_{i}\right)$ is not well defined on $\partial P_{h}$ either. These facts allow us to establish only point-wise convergence of $\lambda_{i}(\mathbf{x})$ for $\mathbf{x} \in P_{h}$. However, $\lambda_{i}(\mathbf{x})$ is piece-wise linear and continuous on $\partial P_{h}$ with $\lambda_{i}\left(\mathbf{p}_{j}\right)=\delta_{i, j}$ (see (4)), but it converges to $h \lambda\left(\mathbf{p}(u), t_{i}\right)=h \delta\left(u-t_{i}\right)$ on $\partial \Omega$. Therefore, the convergence of Wachspress coordinates $\lambda_{i}(\mathbf{x})$ is not uniform over $\bar{\Omega}$.

We have shown how the sequence of Wachspress coordinates for the sequence of polygons $P_{h}$ converging to $\Omega$ relates to the 'limit' Wachspress kernel defined on $\Omega$. We now extend these results to barycentric interpolants and mappings.

\subsection{Wachspress interpolants and mappings}

With the same setting $P_{h} \rightarrow \Omega$ as $h \rightarrow 0$ as above, let $f(t)$ be a $C^{2}$ function defined on $\mathbf{p}(t), g(\mathbf{x})$ the Wachspress kernel interpolant (16) of $f(t)$ at the boundary of $\Omega$, and $g_{h}(\mathbf{x})$ the Wachspress interpolant (15) of the discrete values of $f(t)$ at $\mathbf{p}_{i}$ of $P_{h}$.

Theorem 5.1 For $\mathbf{x} \in \Omega$ it holds $g(\mathbf{x})=g_{h}(\mathbf{x})+\mathcal{O}\left(h^{2}\right)$ as $h \rightarrow 0$.

Proof. We need to show that

$$
\sum_{i=1}^{n} w_{i}(\mathbf{x}) f\left(\mathbf{p}_{i}\right) / \sum_{i=1}^{n} w_{i}(\mathbf{x})=\int_{a}^{b} w(\mathbf{x}, t) f(\mathbf{p}(t)) \mathrm{d} t / \int_{a}^{b} w(\mathbf{x}, t) \mathrm{d} t+\mathcal{O}\left(h^{2}\right) .
$$

This is a direct consequence of Lemma 5.2 applied separately to the numerator and denominator of this equation.

In other words, the sequence of Wachspress interpolants $g_{h}(\mathbf{x})$ converges to the Wachspress kernel interpolant $g(\mathbf{x})$ as $P_{h}$ tends to $\Omega$. The order of convergence is two.

We now derive a similar result for barycentric mappings. Let $\Psi$ be a strictly convex domain, $\mathbf{q}(t)$ a parametrisation of its boundary curve. Let $Q_{h}$ be a sequence of inscribed polygonal approximations of $\Psi$ in correspondence to $P_{h}$; see Fig. 6 for an example. We denote $\mathbf{f}(\mathbf{x}): \Omega \rightarrow \Psi$ the mapping (18) defined by the Wachspress kernel and $\mathbf{f}_{h}(\mathbf{x}): P_{h} \rightarrow Q_{h}$ the Wachspress mapping (17).

Theorem 5.2 For $\mathbf{x} \in \Omega$ it holds $\mathbf{f}(\mathbf{x})=\mathbf{f}_{h}(\mathbf{x})+\mathcal{O}\left(h^{2}\right)$ as $h \rightarrow 0$.

Proof. The same approach as in Theorem 5.1 gives the result, this time using a vector version (coordinate-wise) of Lemma 5.2.

Thus, a similar result for interpolants also applies to mappings, with the same convergence order.

Remark 5.2 The above approach establishes only point-wise convergence (cf. Remark 5.1) of Wachspress interpolants and mappings to their limit counterparts for $\mathrm{x} \in \Omega$. The result extends to the boundary via the interpolation property of the interpolants and the continuity of the mappings; see Section 4.

However, our numerical experiments presented in Section 7 suggest uniform convergence. This leads us to the conjecture that Wachspress interpolants and mappings converge uniformly to the corresponding limits given by the Wachspress kernel over $\bar{\Omega}$.

Conjecture 1 There exist constants $F$ and $G$ independent of $\mathrm{x}$, such that

$$
\left|g(\mathbf{x})-g_{h}(\mathbf{x})\right| \leq G h^{2} \quad \text { and } \quad|| \mathbf{f}(\mathbf{x})-\mathbf{f}_{h}(\mathbf{x}) \| \leq F h^{2}
$$

for all $\mathbf{x} \in \bar{\Omega}$ as $h \rightarrow 0$. 

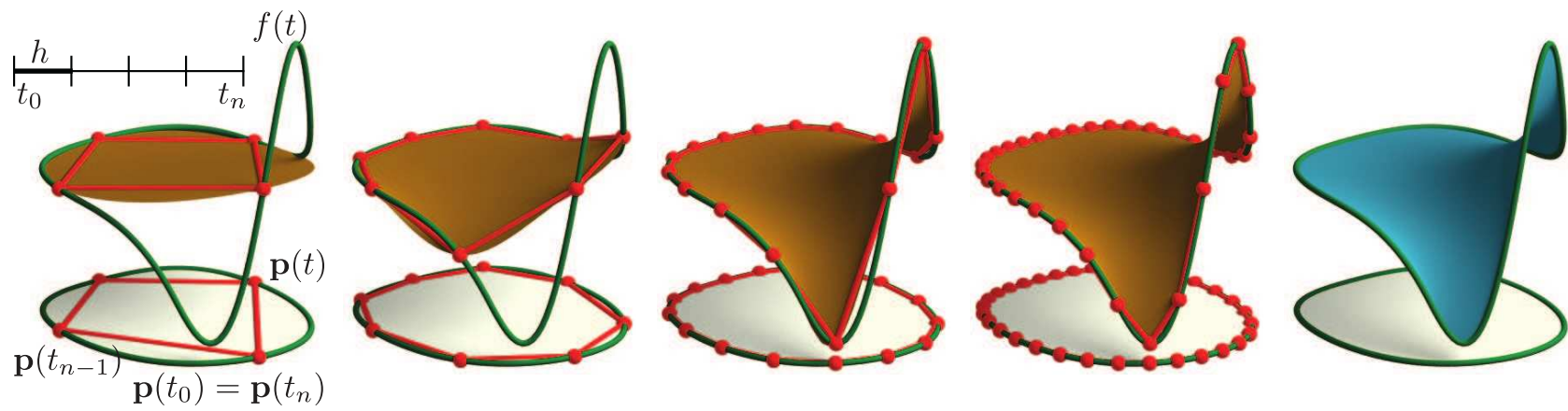

Figure 4: From left to right: A sequence of Wachspress interpolants for given function values at vertices of $n$-sided polygons ( $n=4,8,16,32)$, inscribed into a smooth domain with boundary curve $\mathbf{p}(t)$ is shown. The parameter domain of $\mathbf{p}$ is sampled uniformly with step size $h=\left(t_{n}-t_{0}\right) / n$. Right: The limit smooth case, i.e., the Wachspress kernel interpolant.

The results stated in Theorems 5.1 and 5.2 imply that only relatively coarse polygonal approximations of smooth domains are required to obtain sufficiently good approximations of barycentric interpolants and mappings over smooth domains. We address this further in Section 7.

Our results also suggest that barycentric coordinates could be combined with an interpolatory subdivision scheme, such as the 4-point scheme [2]. This would be a typical scenario in graphics applications since only a few subdivision steps are used to achieve pixel level precision. Quadratic convergence order then guarantees that the corresponding barycentric interpolants and mappings in the discrete case form sufficiently good approximations of those produced by the limit barycentric kernel.

\section{Mean value coordinates}

We have answered the question of convergence of barycentric coordinates in the Wachspress case, but one can naturally ask the same question about other types of barycentric coordinates. Especially coordinates that are not restricted to convex polygons are of high interest.

As a prime example, mean value coordinates $[4,8]$ are, via (5), defined by

$$
w_{i}(\mathbf{x})=\frac{r_{i+1} A_{i-1}-r_{i} B_{i}+r_{i-1} A_{i}}{A_{i-1} A_{i}},
$$

where $r_{i}=\left\|\mathbf{p}_{i}-\mathbf{x}\right\|$. The smooth counterpart, the mean value kernel [10], is given by

$$
w(\mathbf{x}, t)=\frac{(\mathbf{p}(t)-\mathbf{x}) \times \mathbf{p}^{\prime}(t)}{\|\mathbf{p}(t)-\mathbf{x}\|^{3}}
$$

in combination with (13). The curve $\mathbf{p}(t)$ is now assumed to be at least $C^{1}$.

Our numerical tests (see Section 7) suggest that, in terms of convergence, mean value coordinates exhibit the same behaviour as Wachspress coordinates: the convergence of mean value interpolants and mappings is quadratic and uniform and the limit is governed by the mean value kernel. In other words, Conjecture 1 applies in the mean value case as well.

We remark that one could follow our approach also in the mean value setting. However, due to the distance terms $r_{i}$, it has proved difficult to modify Lemmata 5.1 and 5.2 to accommodate the mean value weights. This challenge, combined with proving uniform convergence, remains as future work.

\section{Examples and numerical results}

In this section we present several examples where barycentric coordinates are applied and, due to quadratic convergence, easily replace more computationally demanding barycentric kernels.

Boundary value interpolation is one of the fundamental interpolation problems; see Figs. 4 and 5, where function values are known on the smooth boundary of a domain and function values at interior points need to be estimated. 
Wachspress

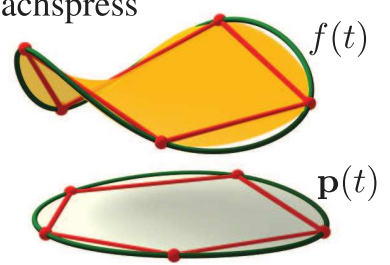

Mean value

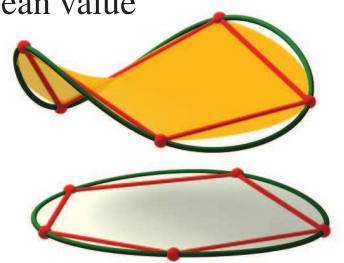

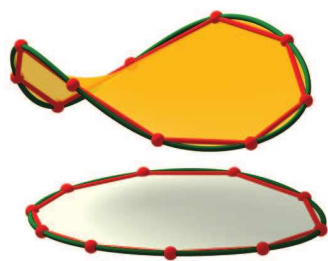
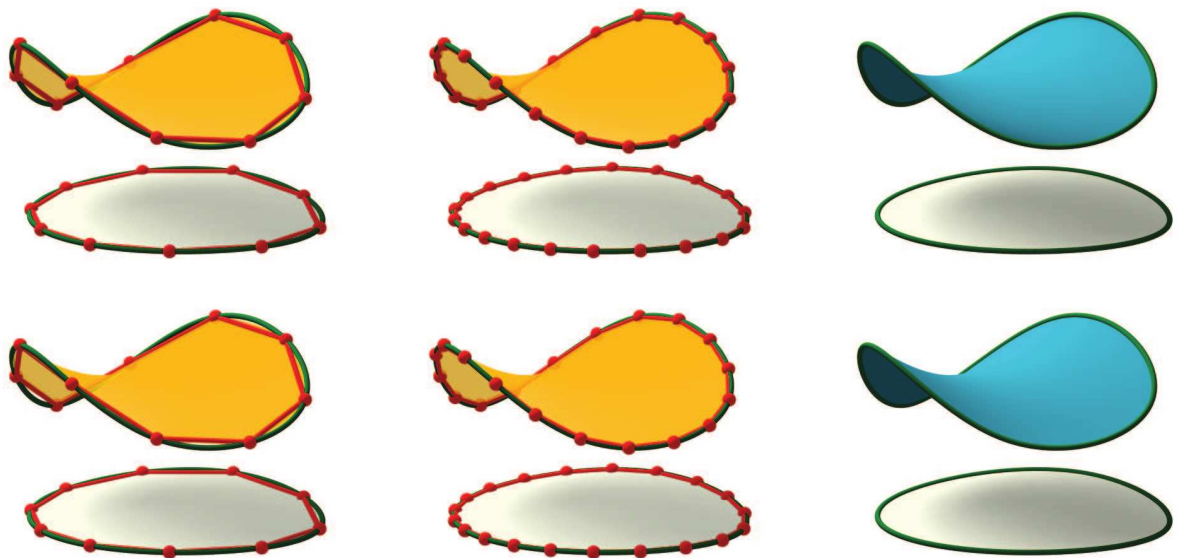

Figure 5: Convergence of barycentric interpolants. A smooth boundary curve $\mathbf{p}(t)$ and a scalar function $f(t)$ are given. From left to right: A sequence of polygons $P_{h}$ inscribed into $\mathbf{p}(t)$ for $n=5,10,20$ with their corresponding barycentric interpolants (yellow) is shown. Right: The limit using the Wachspress kernel and the mean value kernel.
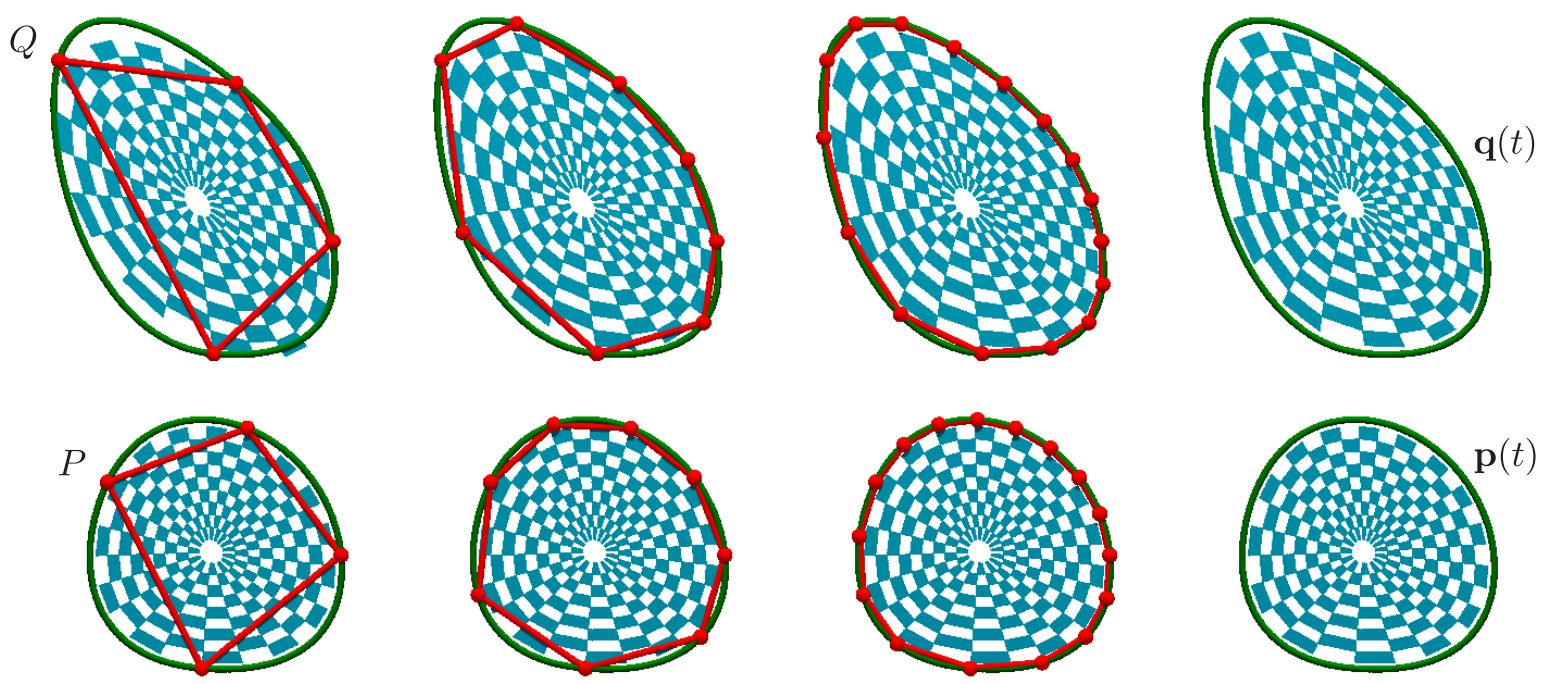

Figure 6: A Wachspress barycentric mapping is defined by a convex source polygon $P$ (bottom) and an image polygon $Q$ (top) and obeys (17). Polygons $P$ and $Q$ (red) are obtained by uniformly sampling the common parameter domain of the smooth boundary curves $\mathbf{p}$ and $\mathbf{q}$. Right: The limit case where the mapping is governed by the Wachspress kernel; see (18).

Another area of applications are deformations using barycentric mappings; see Fig. 6 . The deformation of an object contained inside a smooth domain is governed by the deformation of the boundary curve. The example in Fig. 7 shows a sequence of discrete (polygonal) barycentric deformations applied to font letters. The sequence very quickly captures the features of the shape of the limit case. Note that the image boundary curve $\mathbf{q}$ can be non-convex.

Yet another application comes from the graphics and computer vision community where one needs to estimate a pixel's colour from the colour information at the surrounding pixels; see e.g. [7]. Typically, this is achieved by averaging the values of eight neighbouring pixels, but one may easily apply different blending schemes, in particular Wachspress and/or mean value blends, taking into account different pixel neighbourhoods. This problem is actually a (component-wise) interpolation problem, considering the fact that colour is defined by three channels, e.g. in RGB colour space. As shown in Fig. 8, a colour chart is defined along a smooth boundary curve and the colours of the interior points are estimated using both Wachspress and mean value kernels, as well as using their polygonal counterparts for various sampling densities.

The Wachspress kernel requires $C^{2}$ boundary curves. If the boundary curve is only $C^{1}$ or, even worse, possesses a 
Table 1: Error data for barycentric interpolants and mappings demonstrating point-wise quadratic convergence. In the first column, $i$ is the subdivision level of the parameter domain of the smooth boundary curve $\mathbf{p}(t)$. The number of vertices of $P_{h}$ is $n$. In the smooth case, $n$ is the number of samples taken in a numerical integration of (15) and $\varepsilon$ is an error estimate of the integral in (13). For interpolation, $\varepsilon_{i}=\left|g(\mathbf{x})-g_{i}(\mathbf{x})\right|$ is the absolute error between the smooth and the discrete interpolant at level $i$. For mappings, $\varepsilon_{i}$ is the Euclidean distance between the image points $\mathbf{f}(\mathbf{x})$ and $\mathbf{f}_{i}(\mathbf{x})$. The point $\mathbf{x}$ is taken as the barycentre of the initial (0-th subdivision level) polygon.

\begin{tabular}{|c||r|r|r|r|r|r|}
\hline \multicolumn{1}{|c||}{ Wachspress } & \multicolumn{3}{|c|}{ Interpolation, Fig. 5 } & \multicolumn{3}{c|}{ Mapping, Fig. 7 } \\
$i$ & $n$ & $\varepsilon_{i}$ & $\frac{\varepsilon_{i}}{\varepsilon_{i+1}}$ & $n$ & $\varepsilon_{i}$ & $\frac{\varepsilon_{i}}{\varepsilon_{i+1}}$ \\
\hline \hline 0 & 5 & 0.684923 & $\mathrm{n} / \mathrm{a}$ & 5 & $1.72 e^{-1}$ & $\mathrm{n} / \mathrm{a}$ \\
\hline 1 & 10 & 0.291728 & 2.3480 & 10 & $2.04 e^{-2}$ & 8.4374 \\
\hline 2 & 20 & 0.097361 & 2.9963 & 20 & $6.74 e^{-3}$ & 3.0322 \\
\hline 3 & 40 & 0.028265 & 3.4445 & 40 & $1.74 e^{-3}$ & 3.8679 \\
\hline 4 & 80 & 0.007278 & 3.8832 & 80 & $4.39 e^{-4}$ & 3.9650 \\
\hline 5 & 160 & 0.001816 & 4.0071 & 160 & $1.11 e^{-4}$ & 3.9597 \\
\hline \hline Smooth & $1.5 \mathrm{M}$ & $\varepsilon=8.25 e^{-8}$ & $5 \mathrm{M}$ & \multicolumn{3}{|c|}{$\varepsilon=5.56 e^{-8}$} \\
\hline
\end{tabular}

\begin{tabular}{|c||r|r|r|r|r|r|}
\hline \multicolumn{1}{|c|}{ Mean value } & \multicolumn{3}{|c|}{ Interpolation, Fig. 5 } & \multicolumn{3}{|c|}{ Mapping, Fig. 7 } \\
$i$ & $n$ & $\varepsilon_{i}$ & $\frac{\varepsilon_{i}}{\varepsilon_{i+1}}$ & $n$ & $\varepsilon_{i}$ & $\frac{\varepsilon_{i}}{\varepsilon_{i+1}}$ \\
\hline \hline 0 & 5 & 0.128432 & $\mathrm{n} / \mathrm{a}$ & 5 & $2.67 e^{-1}$ & $\mathrm{n} / \mathrm{a}$ \\
\hline 1 & 10 & 0.025122 & 5.1122 & 10 & $3.44 e^{-1}$ & 5.7679 \\
\hline 2 & 20 & 0.005284 & 4.7538 & 20 & $9.77 e^{-2}$ & 3.5284 \\
\hline 3 & 40 & 0.001248 & 4.2322 & 40 & $2.44 e^{-2}$ & 3.9971 \\
\hline 4 & 80 & 0.000303 & 4.1085 & 80 & $6.12 e^{-3}$ & 3.9947 \\
\hline 5 & 160 & 0.000072 & 4.2305 & 160 & $1.53 e^{-4}$ & 3.9938 \\
\hline Smooth & $2.0 \mathrm{M}$ & $\varepsilon=9.42 e^{-7}$ & $5.0 \mathrm{M}$ & $\varepsilon=2.80 e^{-6}$ \\
\hline
\end{tabular}

'sharp corner' as shown in Fig. 9, the kernel and consequently the associated interpolant is not well defined. In contrast, the sequence of Wachspress interpolants is perfectly valid, converges to a well-defined limit, and can be easily used.

Note that the domains of interpolants and mappings are $\Omega$, not just $P_{h}$. Trimming to $P_{h}$ is not required since the coordinates seem to be well defined on $\Omega$. This fact, if proved in general, would play an important role in applications when one cannot tolerate 'gaps' between the boundary of a domain and an inscribed approximating polygon.

Tables 1 and 2 summarise numerical measurements of our examples. The data in Table 1 confirm our asymptotic analysis discussed in Theorems 5.1 and 5.2 for Wachspress coordinates and indicate similar behaviour for mean value coordinates. The data presented in the table correspond to the barycentre of $P$. Nevertheless, we obtained similar results for random samples in all our examples as well.

The numerical results presented in Table 2 suggest that the quadratic convergence of interpolants and mappings is uniform in both the Wachspress and mean value cases; see Conjecture 1 and Section 6. We performed this numerical analysis of uniform convergence for all presented examples, including the example with a sharp corner in Fig. 9, with analogous results.

Observe that, in general, the integrals in (13), (16), and (18) cannot be expressed in closed form. Hence all the integrals are computed numerically using the trapezoidal rule. In the smooth case in Table $1, \varepsilon$ is the error bound estimate on the integral in (13), computed as the difference between upper and lower sums. Note that these error estimates depend on $\mathbf{x}$ and also on the parametrisation of the boundary curve(s). 
Table 2: Data indicating uniform convergence of barycentric interpolants and mappings. In the first column, $i$ is the subdivision level. The number of vertices of $P_{h}$ is $n$. For interpolation, $m_{i}$ is the maximum difference between the discrete interpolants at level $i$ and $i-1$ over uniformly distributed samples $\mathbf{x} \in \bar{\Omega}, m_{i}=$ $\max _{\mathbf{x} \in \bar{\Omega}}\left|g_{i}(\mathbf{x})-g_{i-1}(\mathbf{x})\right|$. For mappings, $m_{i}$ is the maximum Euclidean distance between the image points $\mathbf{f}_{i}(\mathbf{x})$ and $\mathbf{f}_{i-1}(\mathbf{x})$ over all sampled $\mathbf{x}$. The relative maximum rate $r_{i}=\frac{m_{i-1}}{m_{i}}$ suggests uniform quadratic convergence.

\begin{tabular}{|c||r|r|r|r|r|r|}
\hline \multicolumn{1}{|c||}{ Wachspress } & \multicolumn{3}{|c|}{ Interpolation, Fig. 5, 15K samples } & \multicolumn{3}{|c|}{ Mapping, Fig. 7, 10K samples } \\
$i$ & $n$ & $m_{i}$ & $r_{i}$ & $n$ & $m_{i}$ & $r_{i}$ \\
\hline \hline 0 & 5 & $\mathrm{n} / \mathrm{a}$ & $\mathrm{n} / \mathrm{a}$ & 5 & $\mathrm{n} / \mathrm{a}$ & $\mathrm{n} / \mathrm{a}$ \\
\hline 1 & 10 & 1.724350 & $\mathrm{n} / \mathrm{a}$ & 10 & 1.00769 & $\mathrm{n} / \mathrm{a}$ \\
\hline 2 & 20 & 0.643086 & 2.68137 & 20 & 0.411167 & 2.45081 \\
\hline 3 & 40 & 0.104120 & 6.17642 & 40 & 0.0840098 & 4.89427 \\
\hline 4 & 80 & 0.010176 & 10.2316 & 80 & 0.0127876 & 6.56963 \\
\hline 5 & 160 & 0.002188 & 4.65091 & 160 & 0.0028052 & 4.55851 \\
\hline 6 & 320 & 0.000545 & 4.00922 & 320 & 0.0007005 & 4.00423 \\
\hline 7 & 640 & 0.000136 & 4.00243 & 640 & 0.0001750 & 4.00122 \\
\hline 8 & 1280 & 0.000034 & 4.00062 & 1280 & 0.0000437 & 4.00031 \\
\hline 9 & 3560 & 0.000008 & 4.00014 & 3560 & 0.0000109 & 4.00003 \\
\hline
\end{tabular}

\begin{tabular}{|c||r|r|r|r|r|r|}
\hline \multicolumn{1}{|c||}{ Mean value } & \multicolumn{4}{|c|}{ Interpolation, Fig. 5, 15K samples } & \multicolumn{3}{|c|}{ Mapping, Fig. 7, 10K samples } \\
$i$ & $n$ & $m_{i}$ & $r_{i}$ & $n$ & $m_{i}$ & $r_{i}$ \\
\hline \hline 0 & 5 & $\mathrm{n} / \mathrm{a}$ & $\mathrm{n} / \mathrm{a}$ & 5 & $\mathrm{n} / \mathrm{a}$ & $\mathrm{n} / \mathrm{a}$ \\
\hline 1 & 10 & 1.58148 & $\mathrm{n} / \mathrm{a}$ & 10 & 2.54462 & $\mathrm{n} / \mathrm{a}$ \\
\hline 2 & 20 & 0.551509 & 2.86754 & 20 & 1.12794 & 2.25598 \\
\hline 3 & 40 & 0.133032 & 4.14574 & 40 & 0.362977 & 3.10748 \\
\hline 4 & 80 & 0.0303171 & 4.38796 & 80 & 0.0900502 & 4.03083 \\
\hline 5 & 160 & 0.00766831 & 3.95356 & 160 & 0.0206094 & 4.36947 \\
\hline 6 & 320 & 0.00192334 & 3.98707 & 320 & 0.00440841 & 4.67491 \\
\hline 7 & 640 & 0.00048118 & 3.99701 & 640 & 0.00104064 & 4.23625 \\
\hline 8 & 1280 & 0.00012031 & 3.99926 & 1280 & 0.000260489 & 3.99495 \\
\hline 9 & 3560 & 0.00003008 & 3.99984 & 3560 & 0.000065136 & 3.99912 \\
\hline
\end{tabular}

\section{Conclusions and future work}

We have derived relations between Wachspress coordinates defined over finer and finer polygons converging to a smooth domain and the Wachspress kernel which is reached in the limit. Based on these relations, we have shown that the corresponding sequences of Wachspress interpolants and mappings converge quadratically to their limit counterparts given by the Wachspress kernel. Several examples were shown to validate our theoretical results and to visually assess the behaviour of the interpolants and mappings when converging from the discrete to the smooth case. Our theoretical and numerical observations allow us to conclude that barycentric kernels in the smooth case can be replaced by discrete barycentric approximations due to the fact that the errors become marginal after only a few refinements. This is theoretically supported by our quadratic convergence proof in the Wachspress case.

Our empirical tests with mean value coordinates suggest convergence of the same order as in the Wachspress case. Moreover, based on our numerical analyses, we have conjectured that the convergence of Wachspress and mean value interpolants and mappings is uniform. Rigorous proofs of these conjectures remain a future challenge.

We have considered only inscribed polygonal approximations of the smooth limit domain. Alternatively, one could consider a convergent sequence of circumscribed, instead of inscribed, polygons. Or more generally still, the sequence of polygons could be given by, for example, recursively applying cubic B-spline subdivision to an initial control polygon, a popular technique for generating $C^{2}$ curves. It is expected that the order and type of convergence would remain unaffected.

Our example with a sharp corner (see Fig. 9) suggests that it should be possible to generalise the definition of the Wachspress kernel to accommodate boundary curves that are not $C^{2}$. Any such generalisation leading to a broader class of interpolants and mappings should, as our results show, be equivalent to the limit of a sequence of interpolants and mappings based on Wachspress coordinates for denser and denser polygons. This is an interesting avenue for future research. 

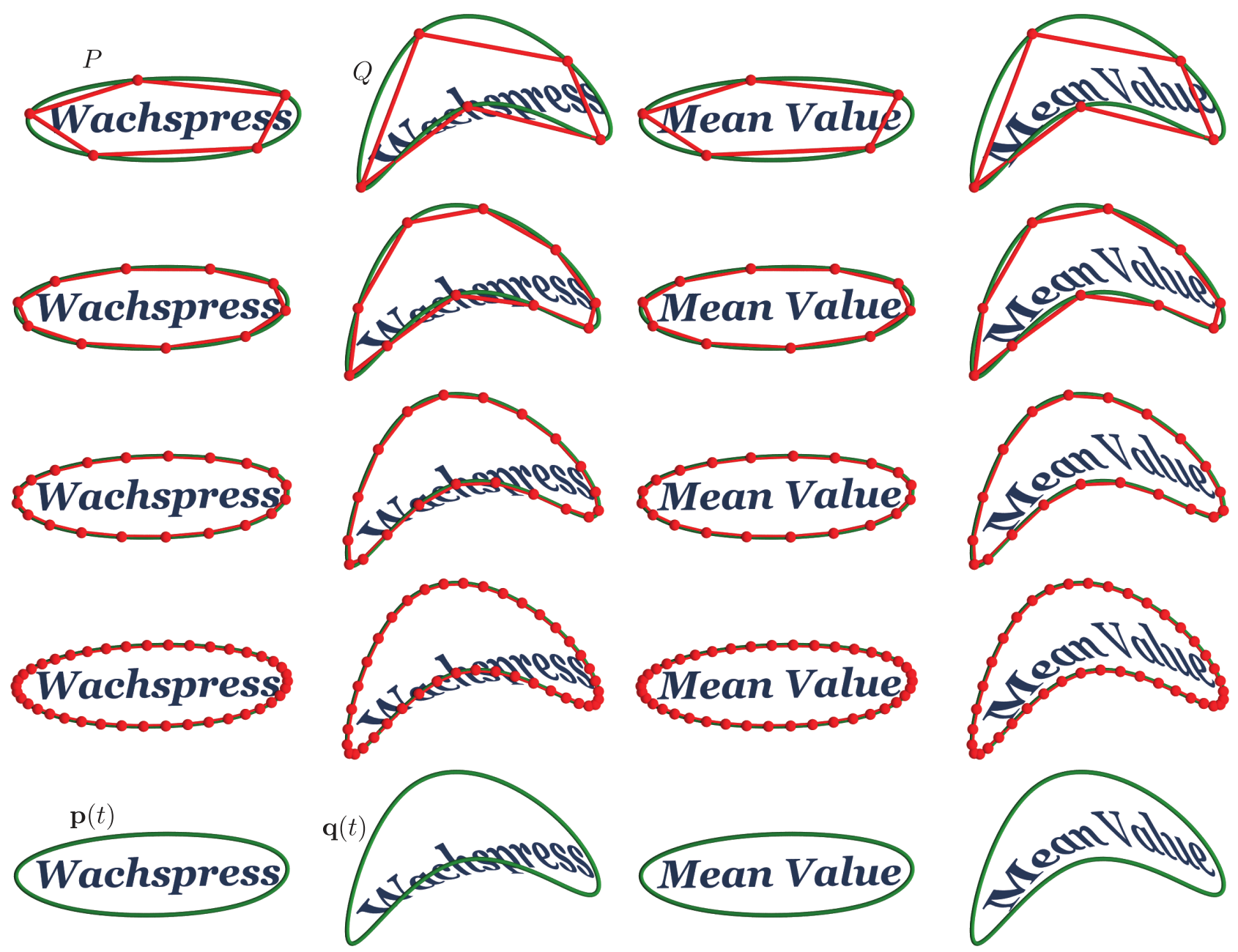

Figure 7: Left: A sequence of barycentric mappings, whose control polygons (red) are sampled on the smooth boundary curves (green), is shown. In the discrete case, the mapping is defined by a convex source polygon $P$ and an image polygon $Q$ and obeys (17). Bottom row: The limit case for smooth boundary curves $\mathbf{p}$ and $\mathbf{q}$, where the mapping is governed by the Wachspress kernel; see (18). Right: Mean value mappings.

\section{Acknowledgements}

This paper was inspired by a conversation with Michael S. Floater. We thank him for his helpful insights. We thank also the anonymous reviewers for their valuable comments. The first author was supported by EPSRC Grant EP/H030115/1.

\section{References}

[1] Dyken, C., Floater, M.S.: Transfinite mean value interpolation. Computer Aided Geometric Design 26(1), 117-134 (2009)

[2] Dyn, N., Levin, D., Gregory, J.A.: A 4-point interpolatory subdivision scheme for curve design. Computer Aided Geometric Design 4(4), 257-268 (1987)

[3] Floater, M., Hormann, K., Kós, G.: A general construction of barycentric coordinates over convex polygons. Advances in Computational Mathematics 24, 311-331 (2006)

[4] Floater, M.S.: Mean value coordinates. Computer Aided Geometric Design 20(1), 19-27 (2003) 

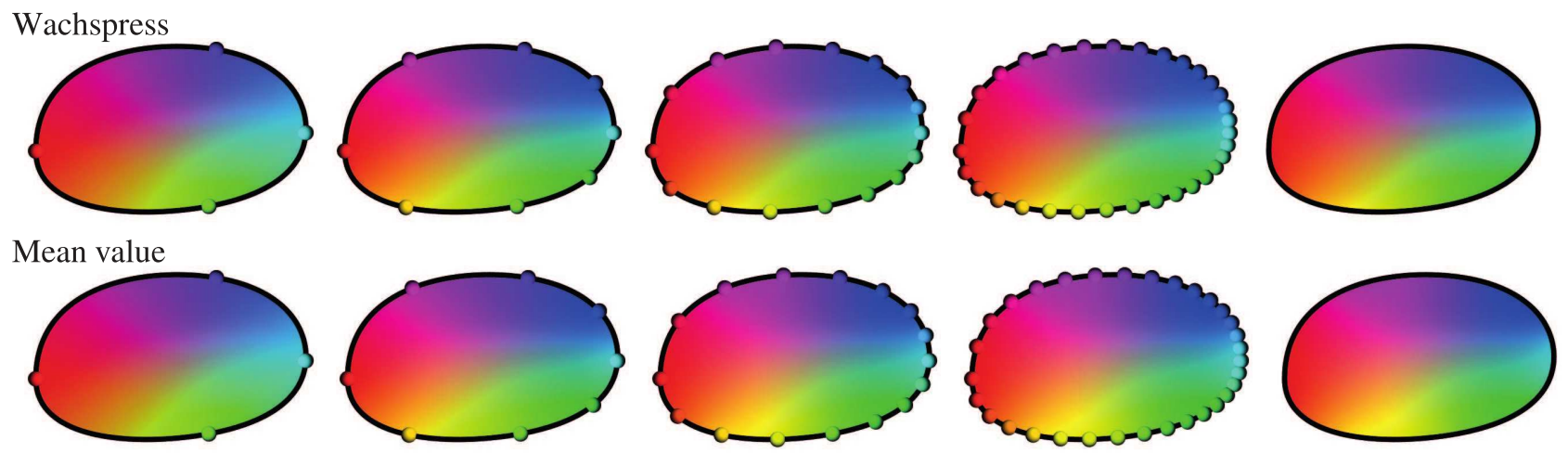

Figure 8: From left to right: A sequence of colour charts given by colour information at boundary points with increasing density of samples. The interior colours are obtained by coordinate-wise interpolation in RGB space. Right: The limit case using the kernels for smooth boundary curves.
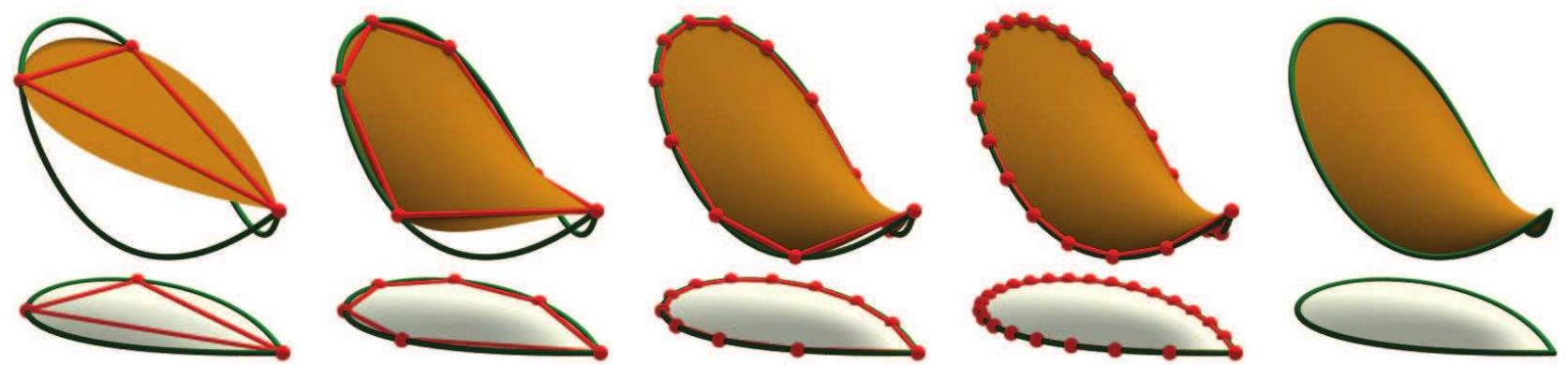

Figure 9: A sequence of Wachspress interpolants defined for polygons with vertices on a boundary curve with a sharp corner is shown. Right: Unlike in the case of a smooth boundary, the Wachspress kernel is not defined. In contrast, the sequence of Wachspress interpolants converges. For visualisation purposes, the interpolant after ten subdivision steps is shown, i.e., the limit is replaced by the discrete case with $3 \cdot 2^{10}$ vertices.

[5] Floater, M.S., Kosinka, J.: Barycentric interpolation and mappings on smooth convex domains. In: Proceedings of the 14th ACM Symposium on Solid and Physical Modeling, SPM '10, pp. 111-116. ACM, New York, NY, USA (2010)

[6] Floater, M.S., Kosinka, J.: On the injectivity of Wachspress and mean value mappings between convex polygons. Advances in Computational Mathematics 32, 163-174 (2010)

[7] Hansen, C.D., Johnson, C.: The Visualisation Handbook. Academic Press (2004)

[8] Hormann, K., Floater, M.S.: Mean value coordinates for arbitrary planar polygons. ACM Transactions on Graphics 25(4), 1424-1441 (2006)

[9] Joshi, P., Meyer, M., DeRose, T., Green, B., Sanocki, T.: Harmonic coordinates for character articulation. ACM Transactions on Graphics 26(3) (2007)

[10] Ju, T., Schaefer, S., Warren, J.: Mean value coordinates for closed triangular meshes. ACM Transactions on Graphics 24(3), 561-566 (2005)

[11] Li, X.Y., Ju, T., Hu, S.M.: Cubic mean value coordinates. ACM Transactions on Graphics 32(4), 98:1-10 (2013)

[12] Meyer, M., Barr, A., Lee, H., Desbrun, M.: Generalized barycentric coordinates on irregular polygons. Journal of Graphics Tools 7(1), 13-22 (2002)

[13] Schneider, T., Hormann, K., Floater, M.: Bijective composite mean value mappings. Computer Graphics Forum 32(5), 137-146 (2013) 
[14] Wachspress, E.L.: A Rational Finite Element Basis. Mathematics in Science and Engineering. Elsevier Science (1975)

[15] Warren, J., Schaefer, S., Hirani, A., Desbrun, M.: Barycentric coordinates for convex sets. Advances in Computational Mathematics 27(3), 319-338 (2007)

[16] Weber, O., Ben-Chen, M., Gotsman, C.: Complex barycentric coordinates with applications to planar shape deformation. Computer Graphics Forum 28(2), 587-597 (2009) 